\title{
Very-high-energy gamma-ray observations of the Type la Supernova SN 2014J with the MAGIC telescopes
}

\author{
M. L. Ahnen ${ }^{1}$, S. Ansoldi ${ }^{2,25}$, L. A. Antonelli ${ }^{3}$, P. Antoranz ${ }^{4}$, C. Arcaro ${ }^{5}$, A. Babic ${ }^{6}$, B. Banerjee ${ }^{7}$, P. Bangale ${ }^{8}$,
} U. Barres de Almeida ${ }^{8,26}$, J. A. Barrio ${ }^{9}$, J. Becerra González ${ }^{10,11,27,36}$, W. Bednarek ${ }^{12}$, E. Bernardini ${ }^{13,28}$, A. Berti ${ }^{2,29}$,

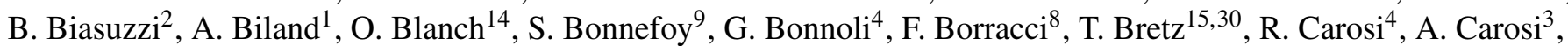
A. Chatterjee ${ }^{7}$, P. Colin ${ }^{8}$, E. Colombo ${ }^{10,11}$, J. L. Contreras ${ }^{9}$, J. Cortina ${ }^{14}$, S. Covino ${ }^{3}$, P. Cumani ${ }^{14}$, P. Da Vela ${ }^{4}$, F. Dazzi ${ }^{8}$, A. De Angelis ${ }^{5}$, B. De Lotto ${ }^{2}$, E. de Oña Wilhelmi ${ }^{16}$, F. Di Pierro ${ }^{3}$, M. Doert ${ }^{17}$, A. Domínguez ${ }^{9}$, D. Dominis Prester ${ }^{6}$, D. Dorner ${ }^{15}$, M. Doro ${ }^{5}$, S. Einecke ${ }^{17}$, D. Eisenacher Glawion ${ }^{15}$, D. Elsaesser ${ }^{17}$, M. Engelkemeier ${ }^{17}$, V. Fallah Ramazani ${ }^{18}$, A. Fernández-Barral ${ }^{14, \star}$, D. Fidalgo ${ }^{9}$, M. V. Fonseca ${ }^{9}$, L. Font ${ }^{19}$, K. Frantzen ${ }^{17}$, C. Fruck ${ }^{8, \star}$, D. Galindo ${ }^{20}$, R. J. García López ${ }^{10,11}$, M. Garczarczyk ${ }^{13}$, D. Garrido Terrats ${ }^{19}$, M. Gaug ${ }^{19}$, P. Giammaria ${ }^{3}$, N. Godinović 6 , D. Gora ${ }^{13}$, D. Guberman ${ }^{14}$, D. Hadasch ${ }^{21}$, A. Hahn ${ }^{8}$, M. Hayashida ${ }^{21}$, J. Herrera ${ }^{10,11}$,

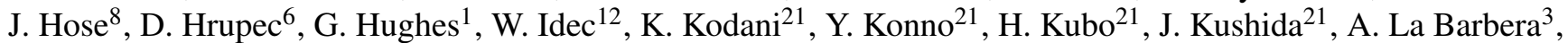
D. Lelas ${ }^{6}$, E. Lindfors ${ }^{18}$, S. Lombardi ${ }^{3}$, F. Longo ${ }^{2,29}$, M. López ${ }^{9}$, R. López-Coto ${ }^{14,31}$, P. Majumdar ${ }^{7}$, M. Makariev ${ }^{22}$, K. Mallot ${ }^{13}$, G. Maneva ${ }^{22}$, M. Manganaro ${ }^{10,11}$, K. Mannheim ${ }^{15}$, L. Maraschi ${ }^{3}$, B. Marcote ${ }^{20}$, M. Mariotti ${ }^{5}$, M. Martínez ${ }^{14}$, D. Mazin ${ }^{8,32}$, U. Menzel ${ }^{8}$, J. M. Miranda ${ }^{4}$, R. Mirzoyan ${ }^{8}$, A. Moralejo ${ }^{14}$, E. Moretti ${ }^{8}$, D. Nakajima ${ }^{21}$, V. Neustroev ${ }^{18}$, A. Niedzwiecki ${ }^{12}$, M. Nievas Rosillo ${ }^{9}$, K. Nilsson ${ }^{18,33}$, K. Nishijima ${ }^{21}$, K. Noda ${ }^{8}$, L. Nogués ${ }^{14}$, S. Paiano ${ }^{5}$, J. Palacio ${ }^{14}$, M. Palatiello ${ }^{2}$, D. Paneque ${ }^{8}$, R. Paoletti ${ }^{4}$, J. M. Paredes ${ }^{20}$, X. Paredes-Fortuny ${ }^{20}$, G. Pedaletti ${ }^{13}$, M. Peresano ${ }^{2}$, L. Perri ${ }^{3}$, M. Persic ${ }^{2,34}$, J. Poutanen ${ }^{18}$, P. G. Prada Moroni ${ }^{23}$, E. Prandini ${ }^{1,35}$, I. Puljak ${ }^{6}$, J. R. Garcia ${ }^{8}$, I. Reichardt ${ }^{5}$, W. Rhode ${ }^{17}$, M. Ribó ${ }^{20}$, J. Rico ${ }^{14}$, T. Saito ${ }^{21}$, K. Satalecka ${ }^{13}$, S. Schroeder ${ }^{17}$, T. Schweizer ${ }^{8}$, A. Sillanpää ${ }^{18}$, J. Sitarek ${ }^{12}$, I. Snidaric ${ }^{6}$, D. Sobczynska ${ }^{12}$, A. Stamerra ${ }^{3}$, M. Strzys 8 , T. Surićc ${ }^{6}$, L. Takalo ${ }^{18}$,

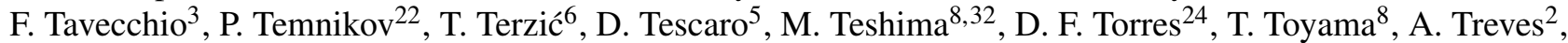

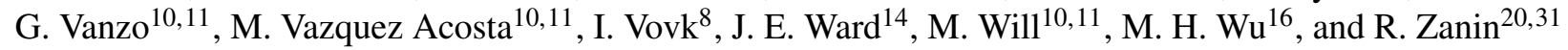

(Affiliations can be found after the references)

Received 24 August 2016 / Accepted 22 February 2017

\section{ABSTRACT}

Context. In this work we present data from observations with the MAGIC telescopes of SN 2014J detected on January 21 2014, the closest Type Ia supernova since Imaging Air Cherenkov Telescopes started to operate.

Aims. We aim to probe the possibility of very-high-energy (VHE; $E \geq 100 \mathrm{GeV}$ ) gamma rays produced in the early stages of Type Ia supernova explosions.

Methods. We performed follow-up observations after this supernova (SN) explosion for five days, between January 27 and February 22014 . We searched for gamma-ray signals in the energy range between $100 \mathrm{GeV}$ and several TeV from the location of SN 2014J using data from a total of $\sim 5.5 \mathrm{~h}$ of observations. Prospects for observing gamma rays of hadronic origin from SN 2014J in the near future are also being addressed.

Results. No significant excess was detected from the direction of SN 2014J. Upper limits at 95\% confidence level on the integral flux, assuming a power-law spectrum, $\mathrm{d} F / \mathrm{d} E \propto E^{-\Gamma}$, with a spectral index of $\Gamma=2.6$, for energies higher than $300 \mathrm{GeV}$ and $700 \mathrm{GeV}$, are established at $1.3 \times 10^{-12}$ and $4.1 \times 10^{-13}$ photons $\mathrm{cm}^{-2} \mathrm{~s}^{-1}$, respectively.

Conclusions. For the first time, upper limits on the VHE emission of a Type Ia supernova are established. The energy fraction isotropically emitted into $\mathrm{TeV}$ gamma rays during the first $\sim 10$ days after the supernova explosion for energies greater than $300 \mathrm{GeV}$ is limited to $10^{-6}$ of the total available energy budget $\left(\sim 10^{51} \mathrm{erg}\right)$. Within the assumed theoretical scenario, the MAGIC upper limits on the VHE emission suggest that SN 2014J will not be detectable in the future by any current or planned generation of Imaging Atmospheric Cherenkov Telescopes.

Key words. gamma rays: general - supernovae: individual: SN 2014J

\section{Introduction}

Type Ia supernovae ( $\mathrm{SNe}$ ) are extremely luminous stellar explosions, which are believed to originate from primary carbonoxygen white dwarfs (WD) in binary systems reaching the Chandrasekhar mass limit of $1.4 M_{\odot}$ (Chandrasekhar 1931).

\footnotetext{
* Corresponding authors: A. Fernández-Barral, e-mail: afernandez@ifae.es; C. Fruck, e-mail: fruck@mpp.mpg.de
}

When this happens, the electron-degenerate core can no longer support the gravitational pressure, leading to an implosion of the progenitor WD. Thereby the temperature grows up to the carbon fusion point, giving rise to a thermonuclear explosion releasing so much nuclear energy $\left(\sim 10^{51} \mathrm{erg}\right.$; Bethe 1993$)$ that no compact remnant is expected. The nature of the companion star is still unclear, although two classical progenitor scenarios have been promoted. Firstly the single-degenerate model, in which the WD 
accretes material from a red giant star (Whelan \& Iben 1973), and secondly the double-degenerate model, in which the explosion is produced by the merging of two WDs (Iben \& Tutukov 1984). Type Ia SNe have been used to provide information on the Galactic chemical evolution (Timmes et al. 1995) and to measure cosmological parameters (e.g. Perlmutter et al. 1999) since they can be used as standard candles thanks to their consistent luminosity (Branch \& Tammann 1992). Still, the evolutionary path that leads to a carbon-oxygen WD which exceeds the Chandrasekhar limit is not well understood yet.

SN 2014J was detected on January 212014 (MJD 56 678) by the UCL Observatory (Fossey et al. 2014) and classified as a Type Ia SN with the Dual Imaging Spectrograph on the ARC $3.5 \mathrm{~m}$ telescope (January 22; Goobar et al. 2014). It is located in the starburst galaxy M 82 at a distance of $3.6 \mathrm{Mpc}$ (Karachentsev \& Kashibadze 2006). Its proximity has granted it the title of the nearest Type Ia SN in the past $42 \mathrm{yr}$ and motivated large multiwavelength follow-up observations from radio to very-high-energy (VHE; $E \geq 100 \mathrm{GeV}$ ) gamma rays.

Deep studies of colour excess and reddening estimation were carried out on SN 2014J. These studies helped to understand the properties of the dust that affects the brightness of the $\mathrm{SN}$ as well as to provide new clues on the progenitors, which are important parameters in the cosmology investigation. Amanullah et al. (2014) presented for the first time a characterization of the reddening of a Type Ia $\mathrm{SN}$ in a full range from $0.2 \mu \mathrm{m}$ to $2 \mu \mathrm{m}$. Their results, with reddening values of $E(B-V) \sim 1.3$ and $R_{V} \sim 1.4$, are compatible with a power-law extinction, expected in the case of multiple scattering scenarios. In the same wavelength band, from UV to near infrared (NIR), Foley et al. (2014) found reddening parameter values of $E(B-V) \sim 1.2$ and $R_{V} \sim 1.4$. In this model, the extinction is explained to be caused by a combination of the galaxy dust and a dusty circumstellar medium. However, although compatible with an extinction law with a low value of $R_{V} \sim 1.4$ and consistent as well with previous mentioned results, Brown et al. (2015), making use of Swift-UVOT data, suggested that most of the reddening is caused by the interstellar dust. Optical and NIR linear polarimetric observations of the source presented in Kawabata et al. (2014) supports the scenario where the extinction is mostly produced by the interstellar dust. These pieces of evidence favour the double-degenerate scenario for SN 2014J, where less circumstellar dust is expected than in cases with a giant companion star. This type of companion is indeed ruled out by several authors as a possible progenitor in SN 2014J, for example, Pérez-Torres et al. (2014), which is the most sensitive study in the radio band of a Type Ia SN, or Margutti et al. (2014) in the X-ray band. The former reported non-detection from the observations performed with eMERLIN and EVN. These results, compared with detailed modelling of the radio emission from the source, allowed them to exclude the single-degenerate scenario in favour of the double-degenerate one with constant density medium of $n \lesssim 1.3 \mathrm{~cm}^{-3}$.

Several authors have speculated about the possibility of SN explosions being able to produce gamma-ray emission at detectable level by current and/or future telescopes. However, these models generally consider Type II SNe due to the strong wind of the progenitors (e.g. Kirk et al. 1995; and Tatischeff 2009). Nevertheless, given the proximity of SN 2014J, this event provides a good exploratory opportunity to probe the possible production of VHE gamma rays during the first days after such an explosion.

In this work, we present the analysis results of SN 2014J observations performed with the MAGIC telescopes.

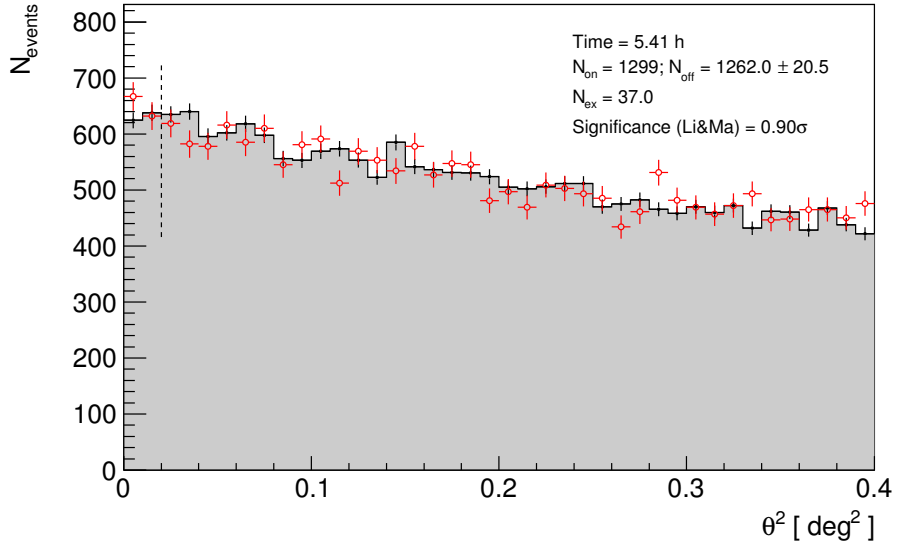

Fig. 1. Distribution of the squared angular distance, $\theta^{2}$, after $5.41 \mathrm{~h}$ of observation between the reconstructed arrival direction of the gammaray candidate events and the position of the source in the camera (red empty circles). The $\theta^{2}$ distribution of the background events (black points) is also displayed. The vertical dashed line at $\theta^{2}=0.02 \mathrm{deg}^{2}$ defines the expected signal region.

\section{Observations and results}

The MAGIC stereo system at the Observatorio del Roque de los Muchachos on the Canary island of La Palma, Spain $\left(28.8^{\circ} \mathrm{N}\right.$, $17.8^{\circ} \mathrm{W}, 2200 \mathrm{~m}$ a.s.1.), consists of two $17 \mathrm{~m}$ Imaging Air Cherenkov Telescopes (IACTs). The MAGIC telescopes reach one of the lowest trigger energy thresholds among current IACTs $(50 \mathrm{GeV})$. The observations were carried out in stereoscopic mode, which means that only shower images seen in both telescopes are recorded and analysed. This mode provides a sensitivity of $0.66 \pm 0.03 \%$ of the Crab Nebula flux in $50 \mathrm{~h}$ of observation for energies above $220 \mathrm{GeV}$ (Aleksić et al. 2016).

SN 2014J was observed under moderate moonlight conditions from January 27 to 29 and on February 1 and 2 under dark-night conditions at medium zenith angles (from $40^{\circ}$ to $52^{\circ}$ ). The MAGIC observations started six days after the first detection by the UCL Observatory because of the adverse weather conditions. The complete data set up to $50^{\circ}(\sim 5.5 \mathrm{~h})$ was used for the analysis given the overall good quality of the data (concerning weather, light conditions and performance of the system).

Figure 1 shows the distribution of squared angular distance $\left(\theta^{2}\right)$ between the reconstructed gamma-ray direction and the position of either SN 2014J (on-source histogram) or the centre of the background control region (off-source histogram). The resulting excess of the on-source histogram over the background from the region, where gamma-ray events from SN 2014J are expected, is compatible with zero excess. The significance computed using Eq. (17) of Li \& Ma (1983) is $0.90 \sigma$.

Upper limits (ULs) on the flux were computed following the Rolke et al. (2005) method for 95\% confidence level (CL), assuming a Gaussian background and a systematic uncertainty of $30 \%$ on the effective area of the instrument (Albert et al. 2008). The spectrum that we assumed was a power-law function, $\mathrm{d} F / \mathrm{d} E \propto E^{-\Gamma}$, with a spectral index of 2.6. Variations of $\sim 20 \%$ in the spectral index produced changes in the integral ULs of less than $5 \%$, and hence small deviations from the used spectral index do not critically affect the reported ULs. The ULs above $300 \mathrm{GeV}$ and $700 \mathrm{GeV}$ for the single-night observations are reported in Table 1 and depicted in Fig. 2.

After $\sim 5.5 \mathrm{~h}$ of observations with the MAGIC telescopes, we established an integral UL on the gamma-ray flux for energies above $300 \mathrm{GeV}$ of $1.3 \times 10^{-12}$ photons $\mathrm{cm}^{-2} \mathrm{~s}^{-1}$ at $95 \% \mathrm{CL}$, 
Table 1. Summary of the MAGIC observations of SN 2014J.

\begin{tabular}{|c|c|c|c|c|c|}
\hline \multicolumn{2}{|c|}{ Date } & \multirow{2}{*}{$\begin{array}{l}\text { Eff. time } \\
{[\mathrm{h}]}\end{array}$} & \multirow{2}{*}{$\begin{array}{c}\mathrm{Zd} \\
{[\mathrm{deg}]}\end{array}$} & \multirow{2}{*}{$\begin{array}{l}\mathrm{UL}(E>300 \mathrm{GeV}) \\
\text { [photons } \mathrm{cm}^{-2} \mathrm{~s}^{-1} \text { ] }\end{array}$} & \multirow{2}{*}{$\begin{array}{l}\mathrm{UL}(E>700 \mathrm{GeV}) \\
\text { [photons } \mathrm{cm}^{-2} \mathrm{~s}^{-1} \text { ] }\end{array}$} \\
\hline [yyyy-mm-dd] & [MJD] & & & & \\
\hline 2014-01-27 & 56684.23 & 0.43 & $47-50$ & $1.03 \times 10^{-11}$ & - \\
\hline 2014-01-28 & 56685.06 & 1.41 & $40-43$ & $2.19 \times 10^{-12}$ & $1.55 \times 10^{-12}$ \\
\hline 2014-01-29 & 56686.09 & 1.30 & $40-42$ & $4.55 \times 10^{-12}$ & $5.97 \times 10^{-13}$ \\
\hline 2014-02-01 & 56689.07 & 0.98 & $40-42$ & $3.14 \times 10^{-12}$ & $9.98 \times 10^{-13}$ \\
\hline 2014-02-02 & 56690.08 & 1.30 & $40-42$ & $3.35 \times 10^{-12}$ & $1.76 \times 10^{-12}$ \\
\hline Total & - & 5.41 & $40-50$ & $1.30 \times 10^{-12}$ & $4.10 \times 10^{-13}$ \\
\hline
\end{tabular}

Notes. From left to right: date of the beginning of the observations, also in modified Julian date (MJD), effective time, zenith angle range and integral ULs at $95 \% \mathrm{CL}$ above 300 and $700 \mathrm{GeV}$. The last row reports the integral ULs derived with the entire data sample. Due to low statistics, no integral UL was computed for energies above $700 \mathrm{GeV}$ for the first day of observations.

which corresponds to $\sim 1.0 \%$ in units of the Crab Nebula flux $(\mathrm{CU})$ in the same energy range. For energies above $700 \mathrm{GeV}$, the integral UL is $4.1 \times 10^{-13}$ photons $\mathrm{cm}^{-2} \mathrm{~s}^{-1}$, corresponding to $\sim 1.1 \% \mathrm{CU}$ at the same CL. Our ULs for $E>700 \mathrm{GeV}$ are already close to the flux from the host galaxy M 82 measured by VERITAS in the same energy range, $\left(3.7 \pm 0.8_{\text {stat }} \pm\right.$ $\left.0.7_{\text {syst }}\right) \times 10^{-13}$ photons $\mathrm{cm}^{-2} \mathrm{~s}^{-1}$ (VERITAS Collaboration et al. 2009), which constitutes an irreducible background for our measurement. Under the hypothesis that M 82 has a gammaray spectrum of $\mathrm{d} F / \mathrm{d} E=3 \times 10^{-16}(E / 1000 \mathrm{GeV})^{-2.5}$ photons $\mathrm{cm}^{-2} \mathrm{~s}^{-1} \mathrm{GeV}^{-1}$, as measured by VERITAS, the expected number of excess events in our observations would be 9.4 , with a $95 \%$ CL lower limit at -6.1 . The observed number of excess events by MAGIC is -4.2 , with an associated $p$-value of $8.4 \times 10^{-2}$, hence consistent with the VHE flux of M 82 measured by VERITAS.

\section{Discussion}

VHE gamma rays are typically secondary products of particle acceleration to $\gtrsim \mathrm{TeV}$ energies, either in hadronic or leptonic processes (Aharonian 2013). The primary particles of such processes may either be protons or electrons. In the first case, the gamma-ray emission is produced by neutral pions from the inelastic collisions between the protons accelerated in the SN and the ambient atomic nuclei. In the latter case, the gamma rays result from inverse Compton (IC) process of the accelerated electrons on the ambient photons. In both cases, the environment plays an important role for the production of VHE gamma-ray radiation. A near and young supernova (approximately one week old) emitting in this energy regime could shed light on the progenitors of these thermonuclear stellar explosions.

Although we did not detect VHE gamma rays right after the explosion, using the known distance of M $82\left(d_{\mathrm{M} 82}=3.6 \mathrm{Mpc}\right.$ Karachentsev \& Kashibadze 2006) and assuming, as before, a spectral index of 2.6, one can convert the measured flux UL into an UL on the power emitted into VHE gamma rays. Therefore, given the integral UL for energies greater than $300 \mathrm{GeV}$, $1.3 \times 10^{-12}$ photons $\mathrm{cm}^{-2} \mathrm{~s}^{-1}$, the resulting UL on the power emitted is of the order of $10^{39} \mathrm{erg} \mathrm{s}^{-1}$. If one now assumes an emission period of the order of ten days, the total energy emitted in VHE gamma rays during this period is smaller than $10^{45} \mathrm{erg}$, which is about $10^{-6}$ of the total available energy budget of the $\mathrm{SN}$ explosion.

Models of the evolution of young supernova remnants (SNRs) can be used to estimate the expected emission from the region in the future. One of the most important parameters to

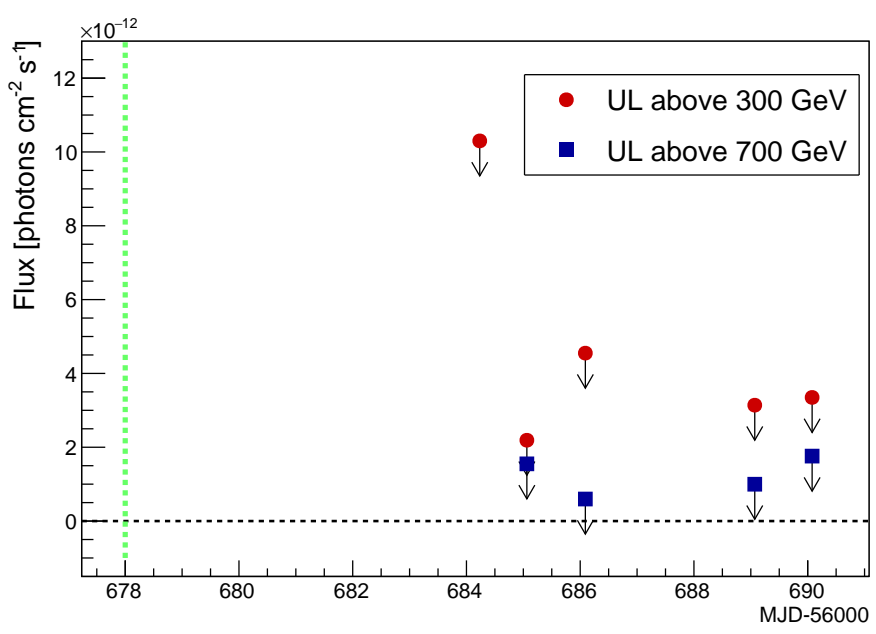

Fig. 2. MAGIC daily integral ULs from the direction of SN 2014J for energies above $300 \mathrm{GeV}$ (red circles) and $700 \mathrm{GeV}$ (blue squares). The integral UL for energies above $700 \mathrm{GeV}$ was not computed for the first night (MJD 56 684) due to low statistics (see also Table 1). The horizontal black dashed line indicates zero flux level and the vertical green line indicates the day of the SN explosion (MJD 56 678), just six days before the beginning of the MAGIC observations.

be assumed is the density profile of the $\mathrm{SN}$ ejecta. In this work, we considered a simple power-law density profile, which allows us to use the Dwarkadas (2013) model to obtain an analytic solution for the estimated flux. Other density profiles have been used in the literature: Models such as W7 or WDD1, applied by Nomoto et al. (1984) and Iwamoto et al. (1999), are usually used in Type Ia SN studies, but they are based on the singledegenerate scenario. Dwarkadas \& Chevalier (1998) discussed a possible exponential density profile, which could represent better the SN ejecta structure than the power-law one. However, the exponential profile cannot provide an analytic result as the one assumed in this work, which can give a correct solution within the order of magnitude, as explained in Dwarkadas (2013).

Thus, making use of Eq. (10) in Dwarkadas (2013), one can obtain the time-dependent emission assuming a hadronic origin. Considering only this hadronic origin, we can establish a lower limit on the total gamma-ray radiation. As discussed above, gamma-ray emission can be also expected from IC processes. Nevertheless, purely leptonic scenarios have been studied and discarded by several authors, for example Völk et al. (2008).

The expected flux depends strongly not only on the assumed density structure of the SNR but also on the density profile 
of the surrounding interstellar medium (ISM). As shown by different authors, we can consider double-degenerate scenario in the case of SN 2014J, that is, two WDs progenitors. WDs do not suffer wind-driven mass-loss and therefore, they are not expected to modify the surrounding medium. Nevertheless, different assumptions, from the lack of certainty on the progenitors, have also been studied (see e.g. Dwarkadas 2000). We can then assume that the Type Ia SN explosion took place in a uniform density medium. In this work, we used a density of $n=2.2 \times 10^{-24} \mathrm{~g} / \mathrm{cm}^{3}$, based on Pérez-Torres et al. (2014), assuming that all the content in the host galaxy of our source, M 82, stems from the neutral hydrogen, $\mathrm{H}_{\mathrm{I}}$. This homogeneous medium assumption leads to an increasing flux emission, above a certain gamma-ray energy, with time in the free-expansion SNR stage, as shown below in the expression given by Dwarkadas (2013):

$F_{\gamma}(>1 \mathrm{TeV}, t)=\frac{3 q_{\gamma} \xi\left(\kappa C_{1}\right)^{5} m^{3}}{6(5 m-2) \beta \mu m_{p} d^{2}} n^{2} t^{5 m-2}$,

where the assumed parameters in this work are

- $q_{\gamma}=1 \times 10^{-19} \mathrm{~cm}^{3} \mathrm{~s}^{-1} \mathrm{erg}^{-1} \mathrm{H}$-atom ${ }^{-1}$ (for energies greater than $1 \mathrm{TeV}$ ) is the emissivity of gamma rays normalised to the cosmic ray energy density tabulated in Drury et al. (1994). This value corresponds to a spectral index of 4.6 of the parent cosmic ray distribution, which was selected according to the assumed spectral index in this work, $\Gamma=2.6$;

$-\xi=0.1$ is the fraction of the total SN explosion energy converted to cosmic ray energy, so an efficient cosmic ray acceleration is assumed;

$-\kappa=1.2$ is the ratio between the radius of the forward shock and the contact discontinuity (which separates ejecta and reverse shock);

- $C_{1}=1.25 \times 10^{13} \mathrm{~cm} / \mathrm{s}^{m}$ is referred to as a constant related to the kinematics of the $\mathrm{SN}$. This value is calculated from the relation given by Dwarkadas (2013), $R_{\text {shock }}=\kappa C_{1} t^{m}$. In turn, $R_{\text {shock }}$ is obtained from Eq. (2) in Gabici et al. (2016), by assuming an explosion energy of $10^{51} \mathrm{erg}$, a mass of the ejecta of $1.4 M_{\odot}$ and a ISM density of $1.3 \mathrm{~cm}^{-3}$, whose value is constrained by Pérez-Torres et al. (2014);

$-\beta=0.5$ represents the volume fraction of the already shocked region from which the emission arises;

$-\mu=1.4$ is the mean molecular weight;

- $m_{\mathrm{p}}=1.6 \times 10^{-24} \mathrm{~g}$ is the proton mass;

$-d=3.6 \mathrm{Mpc}$ is the distance to our source;

- $t$ is the elapsed time since the explosion; and

- $m$ is the expansion parameter.

The expansion parameter varies along the free-expansion phase in different ways according to the assumed model for the density structure of the SN ejecta after the explosion. In this work, we make use of the power-law profile with a density profile proportional to $R^{-7}$ (Chevalier 1982), where $R$ is the outer radius of the ejecta. The initial value of the expansion parameter is very unalike depending on the density profile assumed, but in all cases evolve to $m=0.40$ (Dwarkadas \& Chevalier 1998). This limit at 0.40 is constrained by the beginning of the Sedov-Taylor phase.

The expansion parameter for the power-law profile keeps constant at 0.57 in the first years of the free-expansion stage. Given this value, the expected flux above $1 \mathrm{TeV}$ (constrained by the emissivity of gamma rays, $q_{\gamma}$, tabulated in Drury et al. 1994) at the time of the MAGIC observations ( $t=6$ days) from Eq. (1) is $\approx 10^{-24}$ photons $\mathrm{cm}^{-2} \mathrm{~s}^{-1}$. This flux is consistent with the UL at $95 \%$ CL derived from MAGIC data in the same energy range, $2.8 \times 10^{-13}$ photons $\mathrm{cm}^{-2} \mathrm{~s}^{-1}$ and hence, the powerlaw density profile could be considered as a possible model to describe the density structure of SN 2014J, considering all the assumptions and parameters selection discussed above. On the other hand, this model predicts a constant parameter of $m=0.57$ during the first $\sim 300 \mathrm{yr}$, after which it starts dropping gradually (Dwarkadas \& Chevalier 1998). Although the flux keeps increasing with time according to Eq. (1), with this low expansion parameter it will still be about $10^{-21}$ photons $\mathrm{cm}^{-2} \mathrm{~s}^{-1} 100 \mathrm{yr}$ after the SN occurred, which is well below the sensitivity of the current and planned VHE observatories - several orders of magnitude below the sensitivity that the future Cherenkov Telescope Array $\left(\mathrm{CTA}^{1}\right)$ will reach.

\section{Conclusions}

The MAGIC telescopes performed observations of the nearest Type Ia SN in the last decades, SN 2014J. No VHE gamma-ray emission was detected. Integral ULs for energies above $300 \mathrm{GeV}$ and $700 \mathrm{GeV}$ were established at $1.3 \times 10^{-12}$ photons $\mathrm{cm}^{-2} \mathrm{~s}^{-1}$ and $4.1 \times 10^{-13}$ photons $\mathrm{cm}^{-2} \mathrm{~s}^{-1}$, respectively, for a $95 \% \mathrm{CL}$ and assuming a power-law spectrum. The flux UL at $E>300 \mathrm{GeV}$ corresponds to an emission power of $<10^{39} \mathrm{erg} \mathrm{s}^{-1}$ or a total maximal emitted VHE gamma-ray energy during the observational period - approximately ten days - of $<10^{45} \mathrm{erg}$, which is about $10^{-6}$ times the total energy budget of a Type Ia SN explosion $\left(\sim 10^{51} \mathrm{erg}\right)$. Following Dwarkadas (2013) model for hadronic gamma-ray flux, a power-law density profile proportional to $R^{-7}$ is consistent with our ULs, although, due to the uncertainties in several parameters, this cannot exclude other, more sophisticated, theoretical scenarios. Assuming this SN density profile and a constant density medium, we can estimate an expected emission from the region of the source of $\approx 10^{-24}$ photons $\mathrm{cm}^{-2} \mathrm{~s}^{-1}$. Following these assumptions, this flux would not increase enough in a near future to be detectable by any current or future generation of IACTs, as CTA.

Acknowledgements. We would like to thank the Instituto de Astrofísica de Canarias for the excellent working conditions at the Observatorio del Roque de los Muchachos in La Palma. The financial support of the German BMBF and MPG, the Italian INFN and INAF, the Swiss National Fund SNF, the ERDF under the Spanish MINECO (FPA2015-69818-P, FPA2012-36668, FPA201568278-P, FPA2015-69210-C6-2-R, FPA2015-69210-C6-4-R, FPA2015-69210C6-6-R, AYA2013-47447-C3-1-P, AYA2015-71042-P, ESP2015-71662-C2-2-P, CSD2009-00064), and the Japanese JSPS and MEXT is gratefully acknowledged. This work was also supported by the Spanish Centro de Excelencia "Severo Ochoa" SEV-2012-0234 and SEV-2015-0548, and Unidad de Excelencia "María de Maeztu" MDM-2014-0369, by grant 268740 of the Academy of Finland, by the Croatian Science Foundation (HrZZ) Project 09/176 and the University of Rijeka Project 13.12.1.3.02, by the DFG Collaborative Research Centers SFB823/C4 and SFB876/C3, and by the Polish MNiSzW grant 745/NHESS-MAGIC/2010/0.

\section{References}

Aharonian, F. A. 2013, Astropart. Phys., 43, 71

Albert, J., Aliu, E., Anderhub, H., et al. 2008, ApJ, 674, 1037

Aleksić, J., Ansoldi, S., Antonelli, L. A., et al. 2016, Astropart. Phys., 72, 76

Amanullah, R., Goobar, A., Johansson, J., et al. 2014, ApJ, 788, L21

Bethe, H. A. 1993, ApJ, 412, 192

Branch, D., \& Tammann, G. A. 1992, ARA\&A, 30, 359

Brown, P. J., Smitka, M. T., Wang, L., et al. 2015, ApJ, 805, 74 Chandrasekhar, S. 1931, ApJ, 74, 81

1 As shown in https://www.cta-observatory.org/science/ cta-performance/ 
Chevalier, R. A. 1982, ApJ, 258, 790

Drury, L. O., Aharonian, F. A., \& Volk, H. J. 1994, A\&A, 287, 959

Dwarkadas, V. V. 2000, ApJ, 541, 418

Dwarkadas, V. V. 2013, MNRAS, 434, 3368

Dwarkadas, V. V., \& Chevalier, R. A. 1998, ApJ, 497, 807

Foley, R. J., Fox, O. D., McCully, C., et al. 2014, MNRAS, 443, 2887

Fossey, S. J., Cooke, B., Pollack, G., Wilde, M., \& Wright, T. 2014, Central Bureau Electronic Telegrams, 3792

Gabici, S., Gaggero, D., \& Zandanel, F. 2016, ArXiv e-prints [arXiv: 1610.07638 ]

Goobar, A., Johansson, J., Amanullah, R., et al. 2014, ApJ, 784, L12

Iben, Jr., I., \& Tutukov, A. V. 1984, ApJS, 54, 335

Iwamoto, K., Brachwitz, F., Nomoto, K., et al. 1999, ApJS, 125, 439

Karachentsev, I. D., \& Kashibadze, O. G. 2006, Astrophysics, 49, 3

Kawabata, K. S., Akitaya, H., Yamanaka, M., et al. 2014, ApJ, 795, L4

Kirk, J. G., Duffy, P., \& Ball, L. 1995, A\&A, 293, 37

Li, T.-P., \& Ma, Y.-Q. 1983, ApJ, 272, 317

Margutti, R., Parrent, J., Kamble, A., et al. 2014, ApJ, 790, 52

Nomoto, K., Thielemann, F.-K., \& Yokoi, K. 1984, ApJ, 286, 644

Pérez-Torres, M. A., Lundqvist, P., Beswick, R. J., et al. 2014, ApJ, 792, 38

Perlmutter, S., Aldering, G., Goldhaber, G., et al. 1999, ApJ, 517, 565

Rolke, W. A., Lopez, A. M., \& Conrad, J. 2005, Nucl. Instr. Meth. Phys. Res. A, 551,493

Tatischeff, V. 2009, A\&A, 499, 191

Timmes, F. X., Woosley, S. E., \& Weaver, T. A. 1995, ApJS, 98, 617

VERITAS Collaboration, Acciari, V. A., Aliu, E., et al. 2009, Nature, 462, 770

Völk, H. J., Ksenofontov, L. T., \& Berezhko, E. G. 2008, A\&A, 490, 515

Whelan, J., \& Iben, Jr., I. 1973, ApJ, 186, 1007

1 ETH Zurich, 8093 Zurich, Switzerland

2 Università di Udine, and INFN Trieste, 33100 Udine, Italy

3 INAF National Institute for Astrophysics, 00136 Rome, Italy

4 Università di Siena, and INFN Pisa, 53100 Siena, Italy

5 Università di Padova and INFN, 35131 Padova, Italy

${ }^{6}$ Croatian MAGIC Consortium, Rudjer Boskovic Institute, University of Rijeka, University of Split and University of Zagreb, 51000 Rijeka, Croatia

7 Saha Institute of Nuclear Physics, 1/AF Bidhannagar, Salt Lake, Sector-1, 700064 Kolkata, India

8 Max-Planck-Institut für Physik, 80805 München, Germany

9 Universidad Complutense, 28040 Madrid, Spain

10 Inst. de Astrofísica de Canarias, 38200 La Laguna, Tenerife, Spain

11 Universidad de La Laguna, Dpto. Astrofísica, 38206 La Laguna, Tenerife, Spain

12 University of Łódź, 90236 Lodz, Poland
${ }^{13}$ Deutsches Elektronen-Synchrotron (DESY), 15738 Zeuthen, Germany

14 Institut de Fisica d'Altes Energies (IFAE), The Barcelona Institute of Science and Technology, Campus UAB, 08193 Bellaterra (Barcelona), Spain

15 Universität Würzburg, 97074 Würzburg, Germany

16 Institute for Space Sciences (CSIC/IEEC), 08193 Barcelona, Spain

17 Technische Universität Dortmund, 44221 Dortmund, Germany

18 Finnish MAGIC Consortium, Tuorla Observatory, University of Turku and Astronomy Division, University of Oulu, 90014 Oulu, Finland

19 Unitat de Física de les Radiacions, Departament de Física, and CERES-IEEC, Universitat Autònoma de Barcelona, 08193 Bellaterra, Spain

${ }^{20}$ Universitat de Barcelona, ICC, IEEC-UB, 08028 Barcelona, Spain

21 Japanese MAGIC Consortium, ICRR, The University of Tokyo, Department of Physics and Hakubi Center, Kyoto University, Tokai University, The University of Tokushima, Japan

22 Inst. for Nucl. Research and Nucl. Energy, 1784 Sofia, Bulgaria

${ }^{23}$ Università di Pisa, and INFN Pisa, 56126 Pisa, Italy

24 ICREA and Institute for Space Sciences (CSIC/IEEC), 08193 Barcelona, Spain

25 Also at the Department of Physics of Kyoto University, 606-8501 Japan

26 Now at Centro Brasileiro de Pesquisas Físicas (CBPF/MCTI), R. Dr. Xavier Sigaud, 150 - Urca, 22290-180 Rio de Janeiro - RJ, Brazil

27 Now at NASA Goddard Space Flight Center, Greenbelt, MD 20771, USA

28 Humboldt University of Berlin, Institut für Physik Newtonstr. 15, 12489 Berlin, Germany

29 Also at University of Trieste, Italy

30 Now at École polytechnique fédérale de Lausanne (EPFL), 1015 Lausanne, Switzerland

31 Now at Max-Planck-Institut fur Kernphysik, PO Box 103980, 69029 Heidelberg, Germany

32 Also at Japanese MAGIC Consortium, Japan

${ }^{33}$ Now at Finnish Centre for Astronomy with ESO (FINCA), 21500 Turku, Finland

34 Also at INAF-Trieste and Dept. of Physics \& Astronomy, University of Bologna, 40126 Bologna, Italy

35 Also at ISDC - Science Data Center for Astrophysics, 1290 Versoix (Geneva), Switzerland

36 Now at Department of Physics and Department of Astronomy, University of Maryland, College Park, MD 20742, USA 